\title{
Modeling challenges for high-efficiency visible light-emitting diodes
}

\author{
Francesco Bertazzi ${ }^{1,2}$, Stefano Dominici ${ }^{1}$, Marco Mandurrino ${ }^{1}$, Dipika Robidas ${ }^{1,3}$, Zhou Xiangyu ${ }^{1}$, \\ Marco Vallone ${ }^{1}$, Marco Calciati ${ }^{2}$, Pierluigi Debernardi ${ }^{2}$, Giovanni Verzellesi ${ }^{4}$, Matteo Meneghini ${ }^{5}$, \\ Enrico Bellotti ${ }^{6}$, Giovanni Ghione ${ }^{1}$ and Michele Goano ${ }^{1,2}$ \\ ${ }^{1}$ Dipartimento di Elettronica e Telecomunicazioni, Politecnico di Torino, Torino, Italy \\ ${ }^{2}$ IEIIT-CNR, Politecnico di Torino, Torino, Italy \\ ${ }^{3}$ Crystal Growth Centre, Anna University, Chennai, India \\ ${ }^{4}$ Dipartimento di Scienze e Metodi dell'Ingegneria, Università di Modena e Reggio Emilia, Reggio \\ Emilia, Italy \\ ${ }^{5}$ Dipartimento di Ingegneria dell'Informazione, Università di Padova, Padova, Italy \\ ${ }^{6}$ Department of Electrical and Computer Engineering, Boston University, Boston, MA, USA \\ E-mail: francesco.bertazzi@ polito.it
}

\begin{abstract}
The challenges posed by the numerical modeling of GaN-based light-emitting diodes (LEDs) require the extension of current simulation approaches beyond the semiclassical limits. Any theory hoping to predict the complex carrier transport and optical properties of state-of-the-art III-nitride LEDs should combine a genuine quantum approach with an atomistic description of the electronic structure. Semiclassical discontent notwithstanding, computational considerations have elicited the inclusion of quantum corrections within drift-diffusion approaches. However, the lack of first-principles validation tools has left these quantum models largely untested, at least in the context of LED simulation. It is therefore important to compare the results obtained with currently available commercial numerical simulators, in order to assess the predictive capabilities of the advanced physics-based models complementing the driftdiffusion equations.
\end{abstract}

Index Terms - light-emitting diodes, GaN/InGaN, efficiency droop, Auger recombination, trap-assisted tunneling

\section{INTRODUCTION}

$\mathrm{T}$ he elusive nature of the loss mechanisms occurring in GaNbased light-emitting diodes (LEDs) has turned the debate over droop, the non-thermal decline of the internal quantum efficiency (IQE) at high current densities, into a lively controversy that divides the optoelectronic community. Among the mechanisms invoked to explain droop are Auger recombination, carrier delocalization from composition fluctuations, and carrier leakage from the active region [1]. As of this writing, no droop theory formulated so far has been unambiguously proven theoretically or experimentally. The lack of direct experimental information on critical quantities and the inadequacies of standard simulation approaches have left experimentalists and theoreticians to rely upon models ridden of fitting parameters, thus allowing almost any interpretation [2-4].

Among the difficulties that LED modelers are facing we mention the lack of reliable first-principles models for nonradiative and radiative recombination mechanisms, the inconsistencies in the definitions of some optical and transport parameters when used in experimental and in simulation contexts (e.g., bulk Auger coefficients vs. 2D Auger rates), and the large sensitivity of several key quantities to technological/structural details (interface quality, layer homogeneity, doping and composition profiles, etc.) often inaccessible to an accurate experimental determination [5]. It does not help matters that wide-gap materials exhibit a remarkable excitation-dependent nonlinear optical response due to the intricate interplay between the strong attractive electron-hole Coulomb interaction, which leads to significant excitonic signatures in the optical spectra, and the quantumconfined Stark effect caused by piezoelectric fields.

The possibility for Auger recombination to be the major (if not the sole) cause for droop depends critically on the value of the Auger coefficients. While theoretical studies seem now to agree that - at least for In molar fractions corresponding to blue and green emission - indirect processes dominate Auger recombination in bulk InGaN (the authors of [6] have recently reassessed their bulk calculations [7] to correct the inadvertent omission of a normalization factor and the treatment of the long-range part of the Coulomb interaction), the nature of Auger transitions in confined structures (direct versus phononassisted) is still debated [8,9].

It has been observed that a substantial electroluminescence efficiency reduction occurs when an electron-blocking layer is not employed, a fact that makes room for a narrative on carrier leakage as the cause for droop [10,11]. It should also be noted that the concept of non-equilibrium hot electrons in the context of light emitters is non-conventional, and represents a major departure from semiclassical treatments of carrier-transport based on drift-diffusion (DD) approaches. This new realm is originated from the unique combination of electronic properties of III-nitride light emitters, including large band discontinuities and large polarization fields, that differentiates them from those based on conventional III-V and II-VI semiconductors. One might then suggest that any model not including the treatment of hot electrons, particularly the commercial numerical simulators widely used to model GaN-based LEDs, would be inadequate to the task. The intrinsic limitations of the standard DD simulation framework when used to describe carrier transport in GaN LEDs have elicited the inclusion of semiempirical corrections for mechanisms such as tunneling, carrier 
overflow, ballistic overshoot, nonlocal quantum transport across QWs, and Auger-induced leakage. Unfortunately, these effects are hard to discriminate experimentally, which results in a problematic validation of the corresponding models.

An accurate treatment of vertical carrier transport across the active region would imply to replace the semiclassical Boltzmann picture with genuine quantum approaches based, e.g., on the density-matrix formalism, the Green's function theory, or the Wigner function picture [12]. Moreover, the experience with DD models clearly underscores the need for full-Brillouin-zone approaches [13] to correctly model hot electron effects (and Auger-induced leakage, if relevant). A quantum transport model based on a full-Brillouin-zone atomistic description of the electronic structure accounting for the effects listed above is not available yet; in the following, we will focus our discussion on a few "quantum corrections" currently available in commercial DD-based tools for the design of visible LEDs.

\section{QUANTUM CORRECTIONS}

\section{A. Coupling of DD and Poisson-Schrödinger Equations}

Axiomatic to all semiclassical descriptions of carrier transport across QWs is the separation between unconfined and confined populations, described by three- and two-dimensional (3D, 2D) carrier densities, respectively. The separation of bound and unbound carriers is usually forced by defining an appropriate energetic boundary, e.g., the minimum of the surrounding barrier band edge. The behavior of 3D and 2D populations is fundamentally different, as the different degree of confinement has a profound effect on the scatterings experienced by the carriers. The bound population is represented by localized and stationary states, carrier confinement being considered strong enough so that a fully coherent quantum-mechanical description of localization in the confinement directions appears appropriate. Of course, one cannot expect quantum coherence to be preserved over the entire light-emitting device, whose spatial extension may be of several hundred micrometers. Indeed, coherence is assumed to be completely lost for the unbound population when carrier transport is described within a drift-diffusion (DD) approximation. The two populations are coupled by electrostatics - the solution of the Schrödinger equation has to be reconciled with the Poisson equation - and by the capture of carriers. The latter may be seen as a recombination mechanism for the unbound population or equivalently as a generation term for the bound population [14].

It is interesting to compare commercial simulators implementing the augmented DD discussed above for the calculation of carrier transport across the active region of $\mathrm{GaN}$ LEDs. In the present work, we have used Crosslight APSYS and Synopsys TCAD Sentaurus to simulate the $I(V)$ currentvoltage characteristics of single-QW (SQW) test LED structures manufactured by OSRAM Opto Semiconductors [1517]. Our comparison makes no claim to completeness, also because not all the details of closed-source quantum-corrected
DD implementations are documented. In both simulators, the Poisson and Schrödinger equations (PSE) are solved selfconsistently within a limited portion of the device, where quantum effects are supposed to be more relevant. This approach was first introduced for the modeling of semiconductor lasers in the Minilase simulator [18], and is shared by most present-day codes. However, these codes use different algorithms for determining the extension of the region where the PSE are solved, adopt different boundary conditions for the Schrödinger equation, and use the PSE results in the DD model in subtly different ways inside and outside the QWs. (Additional differences, e.g. in the $\boldsymbol{k} \cdot \boldsymbol{p}$ models and model parameters available to the user, play a critical role in the determination of the emitted optical power spectra, which will not be considered here.) The cumulative effects of these differences can be observed in Fig.1, where the perfect agreement between the $I(V)$ curves obtained (however nontrivially) from classical DD simulations with APSYS and Sentaurus gives place to significant differences at all bias regimes when PSE and DD are coupled. The results of both simulators are also extremely sensitive to the extension of the region where the PSE are solved, which puts into question the predictive capabilities of the PSE-DD approach.

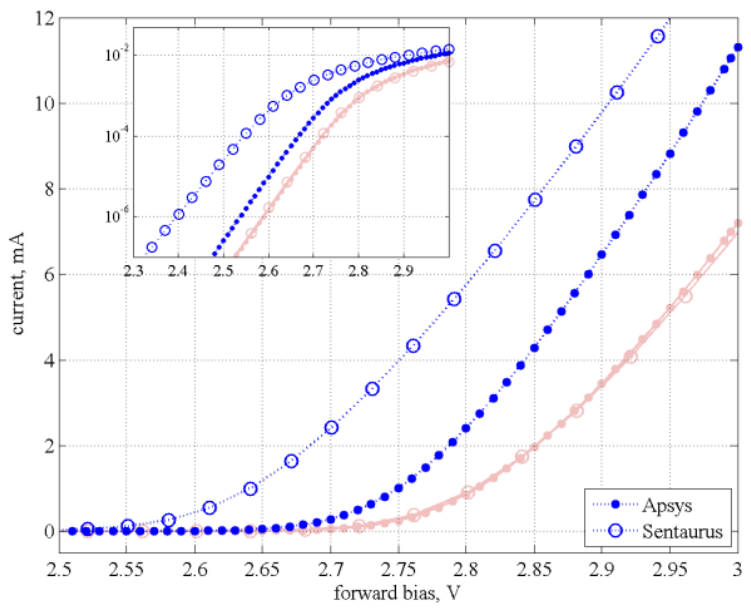

Fig. 1. $I(V)$ characteristics of a SQW test LED structure simulated with Crosslight APSYS and Synopsys TCAD Sentaurus. (The same curves are reported in logarithmic scale in the inset.) While a perfect match is obtained with classical DD simulations (red curves), the inclusion of quantum corrections leads to significant differences at all bias regimes.

\section{B. Inclusion of Trap-Assisted Tunneling in a DD Framework}

Besides acting as SRH recombination centers, the traps located in the LED active region may also assist the tunneling of carriers across it, thus opening additional current leakage channels not accessible by direct band-to-band transitions. The high ideality factor experimentally observed below the optical turn-on of GaN LEDs [19] is the hallmark of trap-assisted tunneling (TAT). Through its signature on the sub-threshold forward-bias characteristics, TAT may represent a useful indicator of the presence of defects and then, in turn, of device growth quality [20] or degradation due to accelerated stress 
testing [21]. But the importance of TAT does not seem to be limited to the sub-threshold regime; for example, reports attempting to peg the efficiency degradation to TAT have been presented by [22-24].

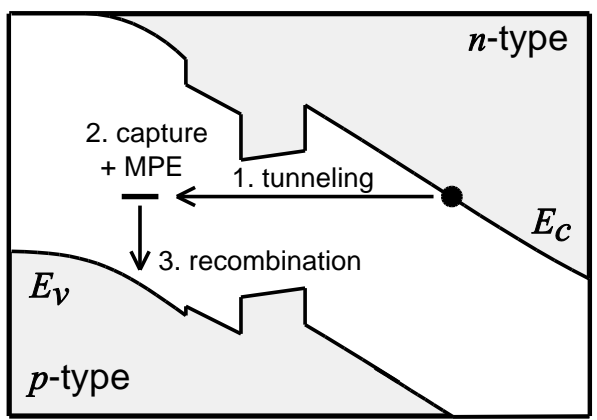

Fig. 2. Schematic description of a TAT process for electrons across the active region of a SQW LED. The tunneling current is determined by the contribution of three different processes (and their respective probabilities): 1 . pure tunneling; 2 . capture by the trap and multiphonon emission (MPE); 3. non-radiative recombination with holes in the valence band in the p-type side. The opposite process can occur for holes towards defects in the $n$-type side of the diode.
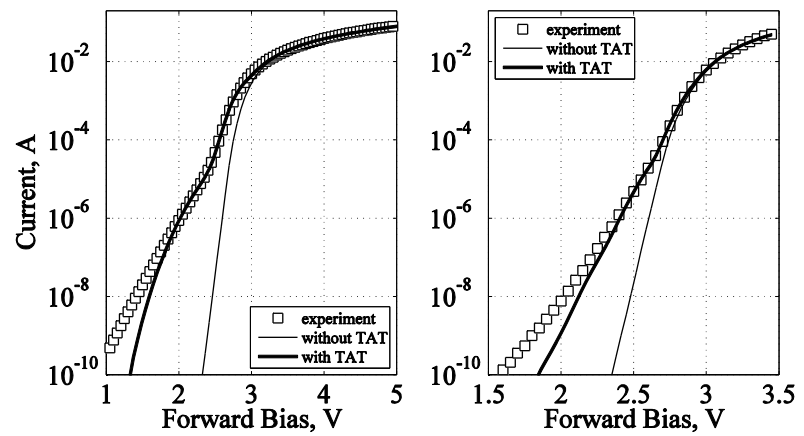

Fig. 3. Room temperature comparisons between measured and simulated characteristics with and without TAT for two SQW test LED structures manufactured by OSRAM (left: $\mathrm{SiC}$ substrate [30], right: Si substrate [15]).

The physics of TAT may be described as the sequence of three events, namely (1) tunneling, (2) capture, and (3) recombination (see Fig. 2). An electron (hole) in the conduction (valence) band tunneling from the $n$ - ( $p$-)side of the diode to the opposite side is captured by a defect. According to multiphonon emission (MPE) theory, the energy of the incoming carrier and the trap energy need not to match for the trapping process to take place (semiclassical theories neglect energy renormalizations of filled traps and assume monomodal phonon emission [22], i.e., a constant phonon dispersion relation). Finally, the capture process is followed by non-radiative recombination. A rigorous modeling of TAT within a genuine quantum mechanical framework such as the density-matrix approach would require an atomistic description of the electronic structure. On the other hand, such model would not be suitable for device-level simulation. Therefore, we regard the three events described above (tunneling, capture and recombination) as a single enhanced recombination process, which we describe with a suitable SRH-like rate [26-28]. The nonlocal nature of this tunneling-enhanced SRH process poses implementation difficulties within local transport models such as DD. Here we follow the approach initially proposed for Si $p$ $n$ junctions [26,27]. A detailed description and theoretical derivation of the TAT model from the basic Hurkx and Schenk descriptions [26,27,29] can be found in [17]. Fig. 3 shows that, by including both electron and hole TAT, we were able to accurately reproduce the electrical characteristics of several InGaN/GaN SQW test LED structures grown on different substrates $[30,15]$.

\section{REFERENCES}

[1] G. Verzellesi, D. Saguatti, M. Meneghini, F. Bertazzi, M. Goano, G. Meneghesso, E. Zanoni, "Efficiency droop in InGaN/GaN blue lightemitting diodes: Physical mechanisms and remedies", J. Appl. Phys. 114(7), 071101 (2013), doi: 10.1063/1.4816434

[2] V. Avrutin, S. A. Hafiz, F. Zhang, Ü. Özgür, E. Bellotti, F. Bertazzi, M. Goano, A. Matulionis, A. T. Roberts, H. O. Everitt, H. Morkoç, "Saga of efficiency degradation at high injection inInGaN light emitting diodes", Turk J. Phys. 38(3), 269-313 (2014), doi: 10.3906/fiz-1407-23

[3] F. Bertazzi, M. Goano, X. Zhou, M. Calciati, G. Ghione, M. Matsubara, E. Bellotti, "Looking for Auger signatures in III-nitride light emitters: A full-band Monte Carlo perspective", Appl. Phys. Lett. 106(6), 061112 (2015), doi: 10.1063/1.4908154

[4] J. Piprek, F. Römer, B. Witzigmann, "On the uncertainty of the Auger recombination coefficient extracted from InGaN/GaN light-emitting diode efficiency droop measurements", Appl. Phys. Lett. 106(10), 101101 (2015), doi: 10.1063/1.4914833

[5] M. Calciati, M. Goano, F. Bertazzi, M. Vallone, X. Zhou, G. Ghione, M. Meneghini, G. Meneghesso, E. Zanoni, E. Bellotti, G. Verzellesi, D. Zhu, C. Humphreys, "Correlating electroluminescence characterization and physics-based models of InGaN/GaN LEDs: Pitfalls and open issues", AIP Adv. 4(6), 067118 (2014), doi: 10.1063/1.4882176

[6] E. Kioupakis, D. Steiauf, P. Rinke, K.T. Delaney, C.G. Van de Walle, "First-principles calculations of indirect Auger recombination in nitride semiconductors" (2014), url: http://arxiv.org/abs/1412.7555

[7] K.T. Delaney, P. Rinke, C.G. Van de Walle, "Auger recombination rates in nitrides from first principles", Appl. Phys. Lett. 94(19), 191109 (2009), doi: 10.1063/1.3133359

[8] F. Bertazzi, X. Zhou, M. Goano, G. Ghione, E. Bellotti, "Auger recombination in InGaN/GaN quantum wells. A full-Brillouin-zone study", Appl. Phys. Lett. 103(8) 081106 (2013), doi: 10.1063/1.4819129

[9] R. Vaxenburg, E. Lifshitz, A. L. Efros, "Suppression of Augerstimulated efficiency droop in nitride-based light emitting diodes", Appl. Phys. Lett. 102(3), 031120 (2013), doi: 10.1063/1.4789364

[10] D. S. Meyaard, G.-B. Lin, J. Cho, E.F. Schubert, H. Shim, S.-H. Han, M.-H. Kim, C. Sone, Y. S. Kim, "Identifying the cause of the efficiency droop in GaInN light-emitting diodes by correlating the onset of high injection with the onset of the efficiency droop", Appl. Phys. Lett. 102(25), 251114 (2013), doi: 10.1063/1.4811558

[11] T. Schulz, A. Nirschl, P. Drechsel, F. Nippert, T. Markurt, M. Albrecht, A. Hoffmann, "Recombination dynamics in InGaN quantum wellsContribution of excited subband recombination to carrier leakage", Appl. Phys. Lett. 105(18), 181109 (2014), doi: 10.1063/1.4901256

[12] F. Rossi, Theory of Electron Transport in Semiconductors. A Pathway from Elementary Physics to Nonequilibrium Green Functions, Sprimger Verlag, Berlin, 2011

[13] X. Zhou, F. Bertazzi, M. Goano, G. Ghione, E. Bellotti, "Deriving $k \cdot p$ parameters from full-Brillouin-zone descriptions: A finite-element envelope function model for quantum-confined wurtzite nanostructures", J. Appl. Phys. 116(03), 033709 (2014), doi: $10.1063 / 1.4890585$

[14] M. Vallone, F. Bertazzi, M. Goano, G. Ghione, "Model for carrier capture time through phonon emission in InGaN/GaN quantum wells", Phys. Status Solidi B 252(5), 971-976 (2015), doi: 10.1002/pssb.201451580

[15] D. Schiavon, M. Binder, M. Peter, B. Galler, P. Drechsel, F. Scholz, "Wavelength-dependent determination of the recombination rate coefficients in single-quantum-well GaInN/GaN light emitting diodes", Phys. Status Solidi B 250(2), 283-290 (2013), doi: 10.1002/pssb. 201248286 
[16] M. Mandurrino, G. Verzellesi, M. Goano, M. Vallone, F. Bertazzi, G. Ghione, M. Meneghini, G. Meneghesso, E. Zanoni, "Physics-based modeling and experimental implications of trap-assisted tunneling in InGaN/GaN light-emitting diodes", Phys. Status Solidi A 212(5), 947953 (2015), doi: 10.1002/pssa.201431743

[17] M. Mandurrino, M. Goano, M. Vallone, F. Bertazzi, G. Ghione, G. Verzellesi, M. Meneghini, G. Meneghesso, E. Zanoni, "Semiclassical simulation of trap-assisted tunneling in GaN-based light-emitting diodes", J. Comp. Electron. 14(2), 444-455 (2015), doi: 10.1007/s10825-015-06753

[18] M. Grupen, K. Hess, "Simulation of carrier transport and nonlinearities in quantum-well laser diodes", IEEE J. Quantum Electron. 34(1), 120140 (1998), doi: 10.1109/3.655016

[19] D. Zhu, J. Xu, A.N. Noemaun, J.K. Kim, E.F. Schubert, M.H. Crawford, D.D. Koleske, "The origin of the high diode-ideality factors in GaInN/GaN multiple quantum well light-emitting diodes”, Appl. Phys. Lett. 94(8), 081113 (2009), doi: 10.1063/1.3089687

[20] J. Kim, Y. Tak, J. Kim, S. Chae, J.K. Kim, Y. Park, “Analysis of forward tunneling current in $\mathrm{InGaN} / \mathrm{GaN}$ multiple quantum well light-emitting diodes grown on $\mathrm{Si}$ (111) substrate", J. Appl. Phys. 114(1), 013101 (2013), doi: 10.1063/1.4812231

[21] L. Liu, J. Yang, D. Teng, S. Qi, G.Wang, "An explanation for invalidity of working currents' derating on improving light-emitting diode devices' reliability", J. Appl. Phys. 114(2), 023102 (2013), doi: 10.1063/1.4813092

[22] R. Nana, P. Gnanachchelvi, M.A. Awaah, M.H. Gowda, A.M. Kamto, Y. Wang, M. Park, K. Das, "Effect of deep-level states on currentvoltage characteristics and electroluminescence of blue and UV lightemitting diodes", Phys. Status Solidi A 207(6), 1489-1496 (2010), doi: $10.1002 /$ pssa.200925596

[23] N.I. Bochkareva, E.A. Zhimov, A.A. Efremov, Y.T. Rebane, R.I. Gorbunov, Y.G. Shreter, "Tunnel-recombination currents and electroluminescence efficiency in InGaN/GaN LEDs”, Semicond. 39(5), 627-632 (2005), doi: 10.1134/1.1923571

[24] N.I. Bochkareva, V.V. Voronenkov, R.I. Gorbunov, A.S. Zubrilov, Y.S. Lelikov, P.E. Latyshev, Y.T. Rebane, A.I. Tsyuk, Y.G. Shreter, "Defect-related tunneling mechanism of efficiency droop in III-nitride light-emitting diodes", Appl. Phys. Lett. 96(13), 133502 (2010), doi: 10.1063/1.3367897

[25] D. Pons, S. Makram-Ebeid, "Phonon assisted tunnel emission of electrons from deep levels in GaAs", J. Physique 40(12), 1161-1172 (1979), doi: 10.1051/jphys:0197900400120116100

[26] G.A.M. Hurkx, D.B.M. Klaassen, M.P.G. Knuvers, F.G. O’Hara, “A new recombination model describing heavy-doping effects and lowtemperature behaviour", in 1989 IEEE international electron devices meeting (IEDM '89), pp. 307-310. Washington (1989), doi: 10.1109/IEDM.1989.74285

[27] G.A.M. Hurkx, D.B.M. Klaassen, M.P.G. Knuvers, "A new recombination model for device simulation including tunneling", IEEE Trans. Electron Devices 39(2), 331-338 (1992), doi: 10.1109/16.121690

[28] K. Sakowski, L. Marcinkowski, S. Krukowski, S. Grzanka, E. LitwinStaszewska, "Simulation of trap-assisted tunneling effect on characteristics of gallium nitride diodes", J. Appl. Phys. 111(12), 123115 (2012), doi: 10.1063/1.4730772

[29] A. Schenk, "A model for the field and temperature dependence of Shockley-Read-Hall lifetimes in silicon”, Solid-State Electron. 35(11), 1585-1596 (1992), doi: 10.1016/0038-1101(92)90184-E

[30] M. Meneghini, N. Trivellin, G. Meneghesso, E. Zanoni, U. Zehnder, B. Hahn, "A combined electro-optical method for the determination of the recombination parameters in InGaN-based light-emitting diodes", $J$. Appl. Phys. 106(11), 114508 (2009), doi: 10.1063/1.3266014 